\title{
COMPARISONS BETWEEN DIFFERENT SPECTRA OF AN ELEMENT IN A BANACH ALGEBRA
}

\author{
LAURA BURLANDO \\ Dipartimento di Matematica dell'Università di Genova \\ Via L.B. Alberti 4 \\ 16132 Genova \\ ITALY
}

(Received June 26, 1990)

\begin{abstract}
In this paper we study the relationships among the spectra of the cosets of an element of a Banach algebra in some quotient algebras. We also characterize the spectrum of any $a \in M$ (where $M$ is an ideal of a Banach algebra with identity and moreover has an identity) in the whole algebra in terms of the spectrum of $a$ in $M$.
\end{abstract}

KEY WORDS AND PHRASES. Banach algebras, spectra, ideals. 1991 AMS SUBJECT CLASSIFICATION CODE. 46H99.

\section{INTRODUCTION.}

Let $L$ be a Banach algebra (that is, a linear associative algebra over either the real field or complex field, endowed with a complete norm such that $\|\boldsymbol{a} b\| \leq\|\boldsymbol{a}\|\|\boldsymbol{b}\|$ for any $\boldsymbol{a}, \boldsymbol{b} \in L$ and $\|\boldsymbol{e}\|=1$ if $L$ has an identity $e$ ). We recall that the vector space $\widetilde{L}=L \times K$ (where $K$ denotes the scalar field) is a Banach algebra (with identity, whether $L$ has an identity or not) with respect to the product defined by

$$
(\boldsymbol{a}, \alpha)(\boldsymbol{b}, \beta)=(\boldsymbol{a} b+\beta \boldsymbol{a}+\alpha \boldsymbol{b}, \alpha \beta) \text { for any }(\boldsymbol{a}, \alpha),(\boldsymbol{b}, \beta) \in \widetilde{L}
$$

and the norm defined by $\|(a, \alpha)\|=\|a\|+|\alpha|$ for any $(a, \alpha) \in \widetilde{L}$. The identity element of $\tilde{L}$ is $(0,1)$ (where $\mathbf{0}$ denotes the null element of $L$ ). Henceforth we shall identify the closed two-sided ideal $\{(\boldsymbol{a}, 0): \boldsymbol{a} \in L\}$ of $\widetilde{L}$ with $L$.

Now let $L$ be a complex Banach algebra with identity $e$. For any $a \in L$, let $\sigma(a)$ denote the spectrum of $a$ with respect to $L$ (where some ambiguity may arise, we shall use the symbol $\sigma^{L}(a)$ instead of $\sigma(a))$. Recently Seddighin ([2]) has proved that $\sigma^{\widetilde{L}}((a, \alpha)) \subset \sigma^{L}(a+\alpha e) \cup\{\alpha\}$ for any $a \in L$ and for any $\alpha \in \mathbb{C}$. Actually, in this paper we show how also the opposite inclusion can be proved, so that the equality $\sigma^{\widetilde{L}}((a, \alpha))=\sigma^{L}(a+\alpha e) \cup\{\alpha\}$ holds (Corollary 6). We derive the equality above from the more general result (Proposition 5) mentioned in the second part of the abstract.

By an ideal of $L$ we shall always mean a two-sided ideal. Let $J_{L}$ denote the set of all proper closed ideals of $L$. For any $a \in L$ and for any $J \in J_{L}$, we denote the spectrum of the coset of $a$ in the quotient algebra $L / J$ by $\sigma_{J}(a)$. We remark that $J_{1} \subset J_{2}$ implies $\sigma_{J 2}(a) \subset \sigma_{J 1}(a)$. Moreover, we have $\sigma_{\{0\}}(a)=\sigma(a)$. We are also concerned here with the relationships among the spectra of 
$a \in L$ in different quotient algebras. In particular, we show that

$$
\left.\sigma_{\left(\cap_{1} \leq k \leq n\right.} J_{k}\right)(a)=U_{1 \leq k \leq n} \sigma_{J k}(a)
$$

(Proposition 1 and following remarks) and $\sigma_{\left(\cup_{J \in C} J\right)^{-}}(a)=\cap_{J \in C} \sigma_{J}(a)$ (where the symbol "-" denotes closure) if $C$ is a chain of $J_{L}$ (Proposition 4).

2. RESULTS.

PROPOSITION 1. Let $L$ be a complex Banach algebra with identity, let $J_{1}, J_{2} \in J_{L}$ and let $a \in L$. Then $\sigma_{J_{1} \cap J_{2}}(a)=\sigma_{J_{1}}(a) \cup \sigma_{J_{2}}(a)$.

PROOF. Let $e$ denote the identity of $L$. Since $J_{1} \cap J_{2} \subset J_{k}$ for any $k=1,2$, it follows that $\sigma_{J_{1}}(a) \cup \sigma_{J_{2}}(a) \subset \sigma_{J_{1} \cap J_{2}}(a)$.

Now we prove that $\sigma_{J_{1} \cap J_{2}}(a) \subset \sigma_{J_{1}}(a) \cup \sigma_{J_{2}}(a)$.

Let $\lambda \in\left(\mathbb{C} \backslash \sigma_{J_{1}}(a)\right) \cap\left(\mathbb{C} \backslash \sigma_{J_{2}}(a)\right)$. Then for any $k=1,2$ there exist $b_{k} \in L$ and $\boldsymbol{u}_{k}, v_{k} \in J_{k}$ such that $b_{k}(\lambda e-a)=e+u_{k}$ and $(\lambda e-a) b_{k}=e+v_{k}$. Consequently,

$$
\left(b_{1}-u_{1} b_{2}\right)(\lambda e-a)=e+u_{1}-u_{1}\left(e+u_{2}\right)=e-u_{1} u_{2}
$$

and

$$
(\lambda e-a)\left(b_{1}-b_{2} v_{1}\right)=e+v_{1}-\left(e+v_{2}\right) v_{1}=e-v_{2} v_{1} .
$$

Since $u_{k}, v_{k} \in J_{k}$ for any $k=1,2, u_{1} u_{2}$ and $v_{2} v_{1}$ belong to $J_{1} \cap J_{2}$. Hence $\lambda e-a$ is both left and right invertible modulo $J_{1} \cap J_{2}$, which implies that $\lambda e-a$ is invertible modulo $J_{1} \cap J_{2}$. Hence $\sigma_{J_{1} \cap J_{2}}(a) \subset \sigma_{J_{1}}(a) \cup \sigma_{J_{2}}(a)$.

We remark that from Proposition 1 it follows that $\sigma_{\left(\cap_{1 \leq k \leq n} J_{k}\right)}(a)=\cup_{1 \leq k \leq n} \sigma_{J_{k}}(a)$ for any $a \in L$ if $J_{1}, \ldots, J_{n} \in J_{L}$.

Now let $S$ be an infinite subset of $J_{L}$. We remark that the inclusion

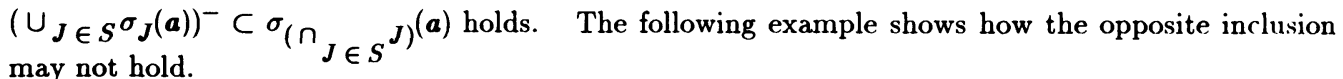

EXAMPLE 2. Let $B$ denote the unit ball of the complex plane, and let $L$ denote the Banach algebra of all complex-valued functions which are continuous on $B^{-}$and holomorphic in $B$. For any $n \in \mathbb{N}$, let $J_{n} \in J_{L}$ be defined by $J_{n}=\left\{f \in L: f\left(q_{n}\right)=0\right\}$ (where $\left\{q_{k}\right\}_{k \in N} \subset B$ has cluster points in $B$ and is not dense in $B$ ). We remark that $\sigma_{J n}(f)=\left\{f\left(q_{n}\right)\right\}$ for any $f \in L$ and for any

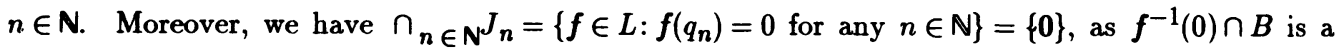
discrete set for any $f \in L \backslash\{0\}$. Thus, if $a \in L$ is defined by $a(x)=x$ for any $x \in B^{-}$, it follows that

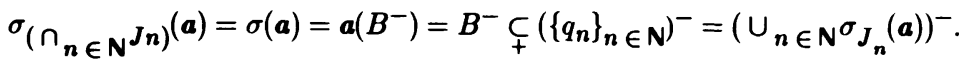

COROLLARY 3. Let $L$ be a complex Banach algebra with identity, let $M \in J_{L}$ and let $a \in M$. Then $\sigma_{J \cap M}(a)=\sigma_{J}(a) \cup\{0\}$ for any $J \in J_{L}$.

PROOF. Since $a \in M$, we have $\sigma_{M}(a)=\{0\}$. Now the result follows immediately from Proposition 1.

It is not difficult to give an example of strict inclusion $\sigma_{J}(a) \subsetneq_{+} \sigma_{J \cap M}(a)$. Let $L$ denote the Banach algebra $C^{2}$ endowed with pointwise product. Then, if we set

$$
M=\{(0, y): y \in \mathbb{C}\}, \quad J=\{(x, 0): x \in \mathbb{C}\} \text { and } a=(0,1),
$$

we have that $a \in M, J \cap M=\{0\}$ and $\sigma_{J}(a)=\{1\} \subsetneq_{\subsetneq}\{0,1\}=\sigma_{J \cap M}(a)$.

We remark that the maximal ideals of a Banach algebra $L$ with identity are closed. Hence, if $C$ is a chain of proper closed ideals of $L$, we have that $\left(\cup_{J \in C} J\right)^{-} \in \mathbb{J}_{L}$. 
PROPOSITION 4. Let $L$ be a complex Banach algebra with identity, and let $C$ be a nonempty chain of proper closed ideals of $L$. Then

for any $a \in L$.

$$
\sigma_{\left(\cup_{J \in C} J\right)^{-}}(a)=\cap_{J \in C^{\sigma}} \sigma_{J}(a)
$$

PROOF. Let $c$ denote the identity of $L$, and let $a \in L$. Since $M \subset\left(\cup_{J \in C} J\right)^{-}$for any $M \in C$, it follows that $\sigma_{\left(\cup_{J \in C} J\right)^{-}}(a) \subset \sigma_{M}(a)$ for any $M \in C$. Hence $\sigma_{\left(\cup_{J \in C} J\right)^{-}}(a) \subset \cap_{J \in C} \sigma_{J}(a)$.

Now we prove the opposite inclusion. We prove that

$$
C \backslash \sigma_{\left(\cup_{J \in C} J\right)^{-}}(a) \subset C \backslash\left(\cap_{J \in C} \sigma_{J}(a)\right)
$$

Let $\lambda \in \mathbb{C} \backslash \sigma_{\left(\cup_{J \in C} J\right)^{-}}(a)$. Then there exist $b \in L$ and $x_{1}, x_{2} \in\left(\cup_{J \in C} J\right)$ such that $b(\lambda e-a)=e+x_{1}$ and $(\lambda e-a) b=e+x_{2}$. Let $M \in C$ be such that there exist $y_{1}, y_{2} \in M$ such that $\left\|x_{j}-y_{j}\right\|<1$ for any $j=1,2$. Then

$$
\left\|b(\lambda e-a)-y_{1}-e\right\|=\left\|x_{1}-y_{1}\right\|<1 \text { and }\left\|(\lambda e-a) b-y_{2}-e\right\|=\left\|x_{2}-y_{2}\right\|<1 .
$$

Since every element of $L$ whose distance from $e$ is less than one is invertible, it follows that $b(\lambda e-a)-y_{1}$ and $(\lambda e-a) b-y_{2}$ are invertible in $L$. Hence there exist $c, d \in L$ such that

$$
c b(\lambda e-a)-c y_{1}=(\lambda e-a) b d-y_{2} d=e .
$$

Since $y_{j} \in M$ for any $j=1,2$, it follows that $\lambda e-a$ is both left invertible and right invertible modulo $M$. Hence $\lambda e-a$ is invertible modulo $M$. Therefore,

$$
\lambda \in \mathbf{C} \backslash \sigma_{M}(a) \subset \mathbf{C} \backslash\left(\cap_{J \in C} \sigma_{J}(a)\right) .
$$

We have thus proved that $\mathbb{C} \backslash \sigma_{\left(\cup_{J \in C}\right)^{-}}(a) \subset \mathbb{C} \backslash\left(\cap_{J \in C} \sigma_{J}(a)\right)$. Hence

$$
\sigma_{\left(\cup_{J \in C}\right)^{-}}(a)=\cap_{J \in C} \sigma_{J}(a) .
$$

We remark that, if $L$ is a complex Banach algebra with identity $e$ and $M$ is a closed subalgebra of $L$, also endowed with an identity $f$, the two identities may not coincide. Moreover, the two identities are necessarily different if $M \in J_{L}$. Nevertheless, the inclusion $\sigma^{L}(a) \subset \sigma^{M}(a) \cup\{0\}$ holds for any $a \in M$ in view of [1], (1.6.12). Since $\sigma^{L}(a+\alpha e)=\sigma^{L}(a)+\alpha$ and $\sigma^{M}(a+\alpha f)=\sigma^{M}(a)+\alpha$ for any $\alpha \in C$, also the inclusion $\sigma^{L}(a+\alpha e) \subset \sigma^{M}(a+\alpha f) \cup\{\alpha\}$ holds for any $a \in M$ and for any $\alpha \in C$. Thus, in particular, the inclusion $\sigma^{\widetilde{L}}((a, \alpha)) \subset \sigma^{L}(a+\alpha e) \cup\{\alpha\}$ for any $a \in L$ and for any $\alpha \in C$ can be deduced.

PROPOSITION 5. Let $L$ be a complex Banach algebra with identity $e$, and let $M$ be a proper ideal of $L$, endowed with an identity $f$. Then $M \in J_{L}$ (which means that $M$ is closed) and $\sigma^{L}(a+\alpha e)=\sigma^{M}(a+\alpha f) \cup\{\alpha\}$ (where we set $\sigma^{M}(0)=\emptyset$ if $M=\{0\}$ ) for any $a \in M$ and for any $\alpha \in \mathrm{C}$.

PROOF. Let $a \in M$. Since $\sigma^{L}(a+\alpha e)=\sigma^{L}(a)+\alpha$ and $\sigma^{M^{-}}(a+\alpha f)=\sigma^{M^{-}}(a)+\alpha$ for any $\alpha \in \mathrm{C}$, it is sufficient to prove that $M$ is closed and $\sigma^{L}(a)=\sigma^{M}(a) \cup\{0\}$.

Since $f$ is the identity of $M$, it follows that $f^{2}=f$. Since the case $M=\{0\}$ is trivial, we can suppose $M \neq\{0\}$, which implies $f \neq 0$. Moreover, since $M$ is a proper ideal of $L$, we have that 
$f \neq e$. Hence $f$ is a proper idempotent of $L$. Then from [1], (1.6.15) it follows that $f L f$ is a closed subalgebra of $L$, with identity $f$, and in addition $\sigma^{L}(a)=\sigma^{f L f}(a) \cup\{0\}$.

Since $M \in J_{L}$ and $f \in M$ it follows that $f L f \subset M$. Moreover, since $f$ is the identity of $M$, we have that $M=f M f \subset f L f$.

We have thus proved that $M=f L f$. Consequently, $M \in J_{L}$ and $\sigma^{L}(a)=\sigma^{M}(a) \cup\{0\}$.

The algebras $L$ and $M$ and the element $a \in M$ introduced in the remark after Corollary 3 provide an example of strict inclusion $\sigma^{M}(a) \subsetneq \sigma^{L}(a)$.

Now let the hypotheses of Proposition 5 hold. For any complex-valued function $h$, holomorphic on an open neighborhood $\triangle$ of $\sigma^{L}(a)$, let $h^{L}(a) \in L$ and $h^{M}(a) \in M$ be defined by

$$
h^{L}(a)=\left(\frac{1}{2 \pi i}\right) \int_{+\partial D} h(\lambda) R^{L}(\lambda, a) d \lambda \text { and } h^{M}(a)=\left(\frac{1}{2 \pi i}\right) \int_{+\partial D} h(\lambda) R^{M}(\lambda, a) d \lambda,
$$

where $R^{L}(\lambda, a)$ (respectively, $R^{M}(\lambda, a)$ ) denotes the inverse of $\lambda e-a$ (respectively, $\lambda f-a$ ) in $L$ (respectively, $M$ ), $+\partial D$ denotes the positively oriented boundary of $D$ and $D$ is an open bounded subset of $C$ such that $\sigma^{L}(a) \subset D \subset D^{-} \subset \triangle, D$ has a finite number of components and $\partial D$ consists of a finite number of simple closed rectifiable curves, no two of which intersect. We recall that the two integrals above are well defined and do not depend on the choice of $D$. From the spectral mapping theorem (see [3], VII, 5.5) and from Proposition 5 it follows that $\sigma^{L}\left(h^{L}(a)\right)=\sigma^{M}\left(h^{M}(a)\right) \cup\{h(0)\}$.

We remark that, actually, the statement above is only seemingly more general than the one of Proposition 5. In fact, for any $\lambda \in C \backslash \sigma^{L}(a)$, we have

$$
\begin{aligned}
(\lambda e-a)\left(R^{M}(\lambda, a)-f / \lambda+e / \lambda\right) & =\lambda R^{M}(\lambda, a)-f+e-a R^{M}(\lambda, a)+a / \lambda-a / \lambda \\
& =(\lambda f-a) R^{M}(\lambda, a)-f+e=e,
\end{aligned}
$$

which implies $R^{L}(\lambda, a)=R^{M}(\lambda, a)-f / \lambda+e / \lambda$. Hence $h^{L}(a)=h^{M}(a)-h(0) f+h(0) e$.

Since any Banach algebra $A$ is a closed proper ideal of $\tilde{A}$, the following result is a consequence of Proposition 5.

COROLLARY 6. Let $L$ be a Banach algebra with identity $e$. Then

$$
\sigma^{\widetilde{L}}((a, \alpha))=\sigma^{L}(a+\alpha e) \cup\{\alpha\} \quad \text { for any } a \in L \quad \text { and for any } \alpha \in C .
$$

Hence the first inclusion proved in [2], Theorem 2.1 can be replaced by an equality.

ACKNOWLEDGEMENT. The author wishes to thank Vladimir Rakočević, who suggested her a shorter proof of Proposition 5.

\section{REFERENCES}

1. RICKART, C.E., General Theory of Banach algebras, Van Nostrand, 1960.

2. SEDDIGHIN, M., Supersets for the spectrum of elements in extended Banach algebras, Internat. J. Math. \& Math. Sci. 12 (1989), 823-824.

3. TAYLOR, A.E. and LAY, D.C., Introduction to Functional Analysis, second edition, Wiley, 1980 . 


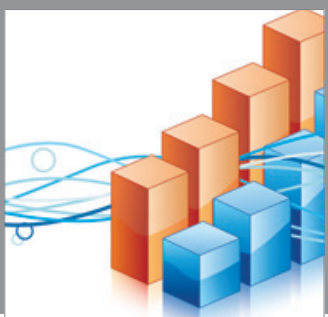

Advances in

Operations Research

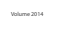

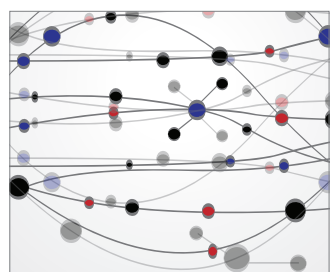

\section{The Scientific} World Journal
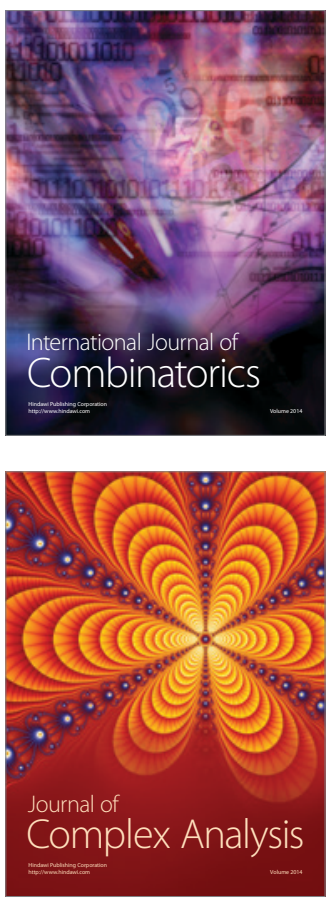

International Journal of

Mathematics and

Mathematical

Sciences
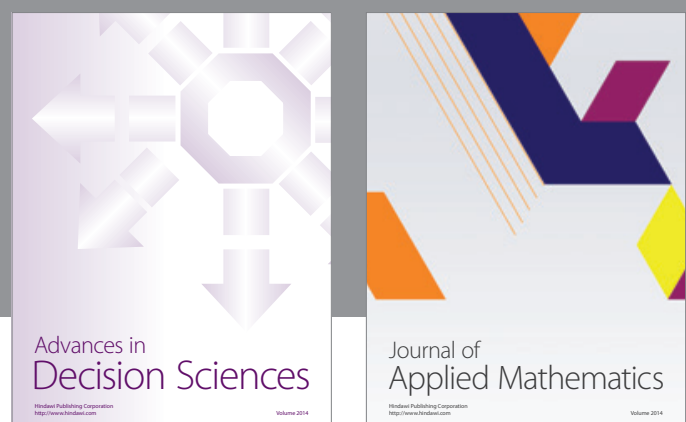

Journal of

Applied Mathematics
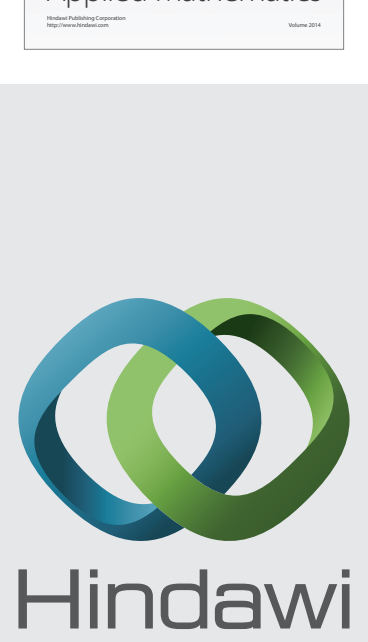

Submit your manuscripts at http://www.hindawi.com
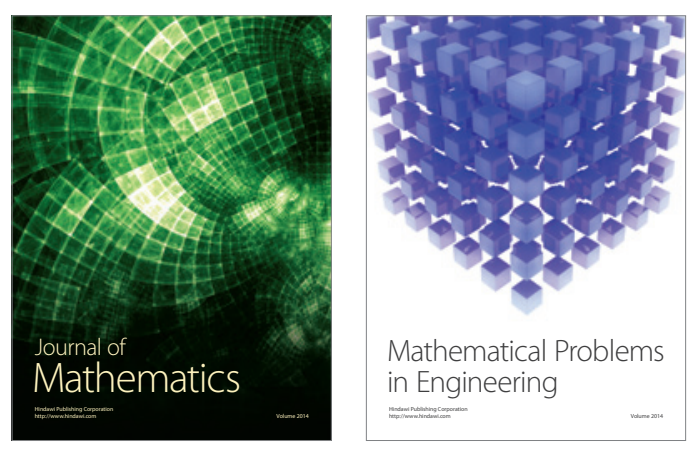

Mathematical Problems in Engineering
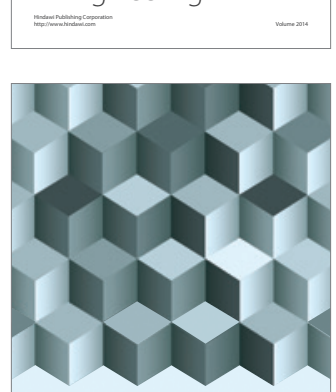

Journal of

Function Spaces
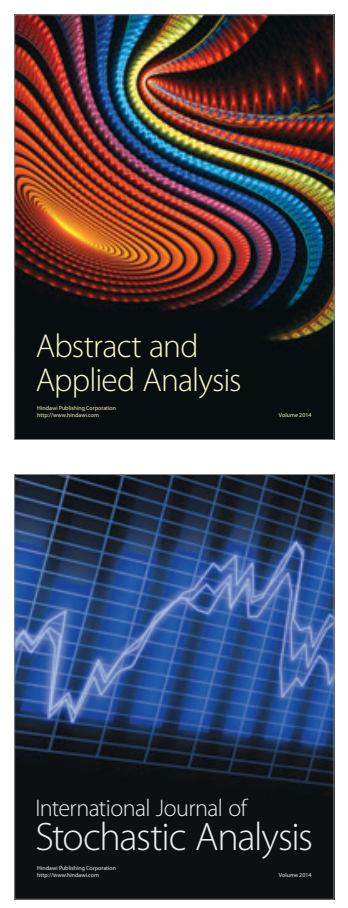

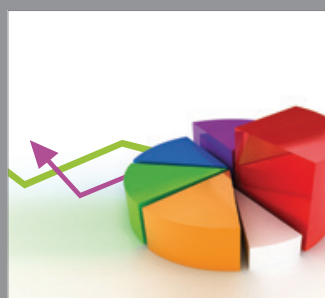

ournal of

Probability and Statistics

Promensencen
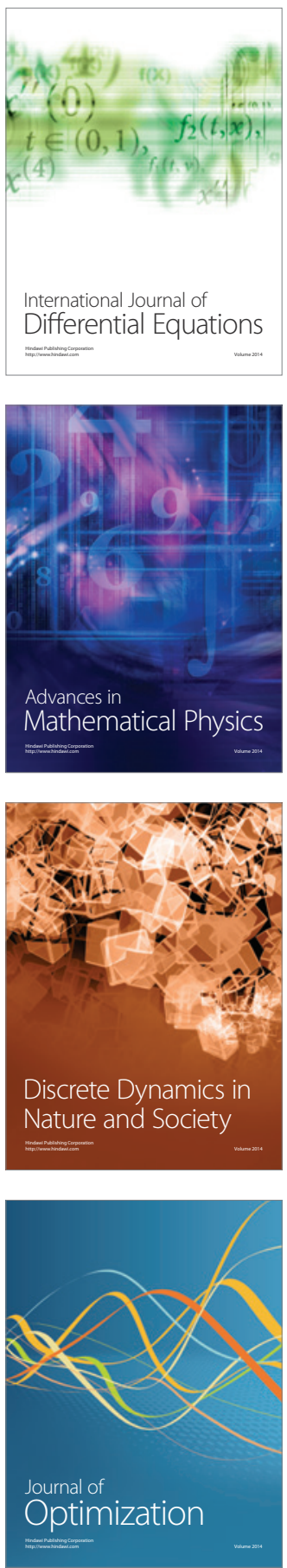